\title{
BMJ Open Chronic Stress protection for postnatal dEpREssioN prEvention (SERENE): a protocol for an exploratory study
}

Dahlia Tharwat, ${ }^{1}$ Marion Trousselard, ${ }^{2}$ Mélanie Balès, ${ }^{3}$ Anne-Laure Sutter-Dallay, ${ }^{3}$ Dominique Fromage, ${ }^{2}$ Elisabeth Spitz, ${ }^{4}$ Dominique Dallay, ${ }^{5}$ Thierry Harvey, ${ }^{1}$ Eric Welter, ${ }^{6}$ Frédéric Coatleven, ${ }^{5}$ Lydie Cherier, ${ }^{5}$ Frédérique Teissèdre, ${ }^{7}$ Jean-Luc Pouly, ${ }^{8}$ Frédéric Dutheil, ${ }^{9}$ Anaïs M Duffaud ${ }^{2}$

To cite: Tharwat $D$, Trousselard M, Balès M, et al. Chronic Stress protection for postnatal dEpREssioN prEvention (SERENE): a protocol for an exploratory study. BMJ Open 2018;8:e018317. doi:10.1136/ bmjopen-2017-018317

- Prepublication history for this paper is available online. To view these files, please visit the journal online (http://dx.doi. org/10.1136/bmjopen-2017018317).

Received 4 July 2017 Revised 13 March 2018 Accepted 15 March 2018
Check for updates

For numbered affiliations see end of article.

Correspondence to Dr Anaïs M Duffaud; anaisduffaud1@gmail.com

\section{ABSTRACT}

Introduction The prevalence of postnatal depression (PND) is significant: reaching up to $20 \%$ in the general population. In mechanistic terms, the risk of PND lies in an interaction between a maternal psychophysiological vulnerability and a chronic environmental context of stress. On the one hand, repetition of stressor during pregnancy mimics a chronic stress model that is relevant to the study of the allostatic load and the adaptive mechanisms. On the other hand, vulnerability factors reflect a psychological profile mirroring mindfulness functioning (psychological quality that involves bringing one's complete and nonjudgemental attention to the present experience on a moment-to-moment basis). This psychological resource is linked to protective and resilient psychic functioning. Thus, PND appears to be a relevant model for studying the mechanisms of chronic stress and vulnerability to psychopathologies. In this article, we present the protocol of an ongoing study (started in May 2017).

Methods and analysis The study is being carried out in five maternities and will involve 260 women. We aim to determine the predictive psychobiological factors for PND emergence and to provide a better insight into the mechanisms involved in chronic stress during pregnancy. We use a multidisciplinary approach that encompasses psychological resources and biophysiological and genetic profiles in order to detect relevant vulnerability biomarkers for chronic stress and the development of PND. To do so, each woman will be involved in the study from her first trimester of pregnancy until 12 months postdelivery. Ethics and dissemination Ethics approval was obtained from the lle de France III Ethics Committee, France (2016A00887-44). We aim to disseminate the findings through international conferences and international peer-reviewed journals.

Trial registration number NCT03088319; Pre-results.

\section{BACKGROUND}

Mounting evidence shows that the perinatal period is a time of increased vulnerability for mood disturbances and the onset of psychiatric disorders. ${ }^{12}$ Among puerperal disorders, baby blues, postnatal depression (PND) and puerperal psychosis are the most frequent. ${ }^{1}$

\section{Strengths and limitations of this study}

- Multidisciplinary approach to postpartum depression: genetic, physiological and psychosocial with three time points: 1, 6 and 12 months postdelivery.

- Identification of early biomarkers that will be good predictors of postpartum depression in order to set up prophylactic intervention.

- Given the way none pathological pregnancies are medically monitored in France (mostly outside of hospitals until the last 2 months of pregnancy), it may be difficult to include 260 participants in their first trimester.

While baby blues appears to be a normal adaptive phenomenon, PND and puerperal psychosis are psychopathologies associated with maternal suffering and have negative consequences for the offspring and the family. PND, the most common childbearing complication, has a prevalence range of 10\%-20\% in Western countries. ${ }^{2}{ }^{3}$ It is described as a non-psychotic depressive episode of mild to moderate severity, beginning in or extending into the first postnatal year. ${ }^{4}$ In the literature, two postpartum peaks have been identified: 4 weeks and 6 months postdelivery. ${ }^{56}$ It is also important to note that PND is the first episode of depression in 6 out of 10 women. ${ }^{7}$

There is no specific diagnostic classification of PND. However, signs and symptoms are described in the Diagnostic and Statistical Manual of Mental Disorders, Fifth Edition (DSM-5) as being similar to those observed in major depressive episodes but with a peripartum onset specifier (either before or after childbirth)..$^{8-10}$ Therefore, the symptoms profile of PND is characterised by major negative effects that are associated with anhedonia (loss of interest in pleasurable activities) and aboulia (eg, behavioural disruption of sleep and appetite). ${ }^{7}$ Such symptoms 
have to be observed for a minimum of 2 weeks and must represent a change from the previous functioning. PND has significant impact on the mother, her partner, the family, mother-infant interactions and on the long-term emotional and cognitive development of the baby. Besides being used for clinical diagnosis, the Edinburgh Postnatal Depression Scale (EPDS) is a screening tool widely used to detect and assess the severity of the depression. ${ }^{11}$

During pregnancy and postpartum periods, the maternal organism undergoes remarkable biological, physical, social and emotional changes. The most studied biological mechanisms are neuroendocrine abnormalities. The hormone withdrawal hypothesis of PND is supported by a number of human and non-human studies: the onset of depressive symptoms is linked to the sudden drop in the concentration of ovarian hormones (estradiol and progesterone). ${ }^{12}{ }^{13}$ Hypothalamo-pituitary-adrenal axis dysfunction also seems to be implicated in PND onset. Corticotropin-releasing hormone, adrenocorticotrophic hormone and cortisol levels increase during pregnancy and up to a few days postdelivery and then normalise within 12 weeks postchildbirth. ${ }^{14}$ Conversely, the involvement of thyroid function in the mother's major depressive episode remains unclear. ${ }^{13}$

Recent neurobiological studies have provided evidence for the involvement of the emotional neuronal circuit in this psychopathology. ${ }^{16}{ }^{17} \gamma$-Aminobutyric acid (GABA) is a potent inhibitory neurotransmitter that has been shown to be altered in PND (decreased inhibition), mainly due to altered receptor functionality. ${ }^{18}$ It is worth noting that GABA functioning is highly dependent on the oxidative status, which is a consequence of an imbalance between the production of free radicals and the failure of the antioxidant defence mechanisms. This dysregulation has been observed in psychopathological disorders such a depression. $^{19}$

Furthermore, three stress-modulator neurotransmitters seem to be directly or indirectly involved in PND: serotonin, dopamine and oxytocin. ${ }^{20}{ }^{21}$ Chronic stress induces metabolic drift associated with reduced serotoninergic and dopaminergic transmission and is probably linked to inflammation as well as depression. ${ }^{20}$ The deleterious effects of stressful situations during the postpartum period (up to 9 weeks) are buffered by endogenous oxytocin, thus protecting women from developing depression. $^{22}$

Other than its unique function during pregnancy, brain-derived neurotrophic factor (BDNF) is a key mediator in neuronal plasticity and in mental disorders such as depression. The neurotrophin hypothesis argues that a stress-induced decrease of BDNF is linked with aberrant neurogenesis and subsequent major depression. This decreased BDNF activity in the brain is correlated with serum/plasma BDNF concentration. Low serum BDNF in late pregnancy is associated with higher depressive symptom ${ }^{23} \mathrm{~s}$.

In addition to hormonal and stress systems, immune dysregulation has also been hypothesised to contribute to
PND. While proinflammatory cytokines decrease during pregnancy, anti-inflammatory cytokines increase. Within the first few hours after delivery, a sudden shift occurs that leads to the installation of a proinflammatory state. ${ }^{24}$ The role of the immune function in the pathophysiology of depression is now clear: higher levels of proinflammatory cytokines are associated with depressive episodes. ${ }^{25}$

Vulnerability to develop PND also arises from genetic polymorphism. ${ }^{26}$ Several lines of evidence suggest that (1) there is a specific genetic polymorphism of major depressive episodes occurring in the first year following birth and (2) clinical severity of the depressive episode is linked to specific genetic variations. ${ }^{27}$ Finally, epidemiological studies have shown an association between gene polymorphisms involved in stress regulation and mood disorders/PND. ${ }^{27} 28$

Socioeconomic and psychological factors are also of great importance in the risk of developing PND. Social context can be either protective (ie, social support) or deleterious (ie, low income) and thus responsible of stress levels during pregnancy. ${ }^{29}$ Epidemiological studies show a link between maternal anxiety and mood symptoms during pregnancy and PND. ${ }^{30}$ Personality aspects (such as neuroticism and low self-esteem), ${ }^{31}$ as well as coping strategies ${ }^{32}$ appear to be key factors in PND vulnerability.

Mindfulness is a psychological quality that involves bringing one's complete attention to the present experience on a moment-to-moment basis and in a particular way: in the present moment and non-judgementally. ${ }^{33}$ This ability is associated with low neuroticism, high self-esteem and efficient coping. ${ }^{34}$ In both healthy individuals and people with medical conditions, mindfulness allows a better regulation of physiological and psychological stress. ${ }^{35} 36$ To the best of our knowledge, mindfulness and major maternal depressive episodes have never been studied. Since this ability is highly beneficial in preventing the development and relapse of depression, ${ }^{37}$ one might expect PND to be less frequent in mindful women.

In light of the gathered evidence (biological, genetic and psychosocial), a hypothesis can be made: the risk of emergence of PND relies on an interaction between a maternal psychobiological vulnerability and a chronic environmental context of stress. PND appears as a relevant model for studying the mechanisms of chronic stress and vulnerability to psychological pathologies.

On the one hand, daily stressors (such as social, familial or work stress) during pregnancy correspond to a natural chronic stress model of several months and thus allows the study of the biological cost of responses to repeated stresses and the adaptive mechanisms leading to the functional drift to psychic dysfunction.

On the other hand, isolated vulnerability factors characterise a mirroring psychological profile of a mindfulness type of functioning. This psychological resource has been associated with resistant or even resilient psychic functioning.

This study aims to follow a cohort of pregnant women from the first trimester of pregnancy to 12 months after 
delivery to determine the predictive psychobiological factors of the emergence of PND.

\section{Objectives}

The primary aim of the study is to determine the predictive psychobiological factors for the emergence of PND. It involves a multidisciplinary approach that will encompass psychological resources and biophysiological and genetic profiles.

The secondary aims are to provide a better insight into the mechanisms involved in chronic stress during pregnancy. We will follow changes in biological, physiological and psychological parameters in order to detect relevant vulnerability biomarkers for chronic stress and the development of PND.

Finally, two exploratory objectives will aim to assess the relevance of neurotrophic factors and genetic polymorphisms in the risk of developing PND.

\section{METHODS}

\section{Patient and public involvement}

Patients and public were not involved in the development of the research question or in the design of the study. Dissemination of the general results (no personal data) would be made on demand.

\section{Trial design and setting}

We aim to undertake a multicentric prospective longitudinal study. The study will take place in hospitals where participants can be included in their first trimester of pregnancy. So far, five hospitals have been involved: Pôle HFME, CHR Mercy, Metz - Maternité GH Diaconesses Croix St Simon, Paris - Centre Aliénor d'Aquitaine, CHU Pellegrin, Bordeaux and Unité FIV, Pôle gynécologie, obstétrique et reproduction, CHU Estaing, Clermont-Ferrand - Centre Hospitalier Sud Francilien, CHSF Evry.

\section{Eligibility criteria}

The women involved in the study will have to meet the following requirements:

\section{Inclusion criteria}

- Before 17 weeks of gestation.

- Pregnancy monitoring planned within one of the facilities involved in the project.

- Full fluent in French.

- Women over 18 years old.

- Covered by the French National Health Service.

\section{Non-inclusion criteria}

- Pathological pregnancy requiring increased medical monitoring.

- Multiple pregnancy.

- Ongoing pathologies at the time of inclusion: (1) immune or endocrine conditions; (2) any psychological disorders (PTSD, depression or anxiety disorders and so on); or (3) neurological pathologies such as multiple sclerosis.
Hormonal or psychotropic drug therapies.

End of the trial

During the study, if a participant meets one the following criteria, her participation will be discontinued:

- Withdrawal of consent.

- Occurrence of a non-inclusion criteria.

- Medical condition interfering with the protocol.

- Death or 'lost-to-follow-up'.

- If a pregnancy becomes 'pathological', the participant will have the choice to continue or to stop her involvement in the project; the pathological event will be recorded in the case report form.

Recruitment started in April 2017 and will last 30 months.

Adverse events and other unintended effects of the study will be reported in compliance with the French public health code.

\section{Intervention: participant timeline}

This study aims to follow pregnant women, civilian or military, from the first trimester of pregnancy up to 12 months postbirth. As shown in figure 1, each woman will have to attend 10 visits: one visit within the first 14 weeks of pregnancy (inclusion visit (VI)), one visit every month during the other two trimesters of pregnancy (VP5 to VP9), one visit within the 48 hours of the birth (VB1) and three visits 2, 6 and 12 months after delivery (VB2, VB3 and VB4, respectively).

The last three VB visits may be performed remotely. The follow-up includes genetic, biological, physiological and/or psychological parameters. During the inclusion visit, participants will receive information on the protocol, provided by competent person (medical doctor or midwife), sign the consent forms (one to participate in the study and one for the genetic analysis) and undergo a complete assessment, which includes psychological questionnaires, measurement of heart rate variability (HRV), and the collection of genetic, hair and blood samples. For the remaining two trimesters of pregnancy, psychological assessments and HRV measurements will be performed monthly, whereas blood sampling will only be done on VP6 and VP8. Within 48 hours of the delivery (VB1), blood and hair will be collected and questionnaires will be completed. The last three visits (VB2, VB3 and VB4) will only require the completion of the psychological questionnaires. Details of the psychological questionnaires and genetic analyses can be found in tables 1 and 2.

\section{Outcomes}

The primary outcome of the study is the identification of predictive psychobiological risks factors for development of PND. PND will be assessed using the EPDS (with a screening cut-off $>11) .{ }^{38}$ The EPDS is a 10 -item self-report questionnaire assessing the symptoms of depression and anxiety. The factors assessed will include psychopathological and positive psychological aspects (see table 1 for detail of the questionnaires used). 


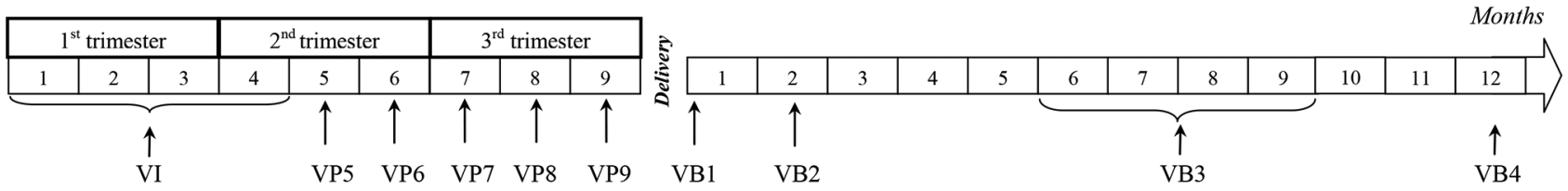

\begin{tabular}{|c|c|c|c|c|c|c|c|c|c|c|}
\hline & \multicolumn{6}{|c|}{ PREGNANCY } & \multicolumn{4}{|c|}{ POST-BIRTH } \\
\hline & \multirow{2}{*}{$\begin{array}{l}\text { Inclusion visit } \\
\quad(\mathrm{VI}) \\
\text { Within the first } \\
19 \text { weeks of } \\
\text { pregnancy }\end{array}$} & \multicolumn{5}{|c|}{$\begin{array}{l}\text { Monthly visit during } \\
\text { trimester } 2 \text { and } 3\end{array}$} & \multicolumn{4}{|c|}{ Post-delivery follow-up visits } \\
\hline & & VP5 & VP6 & VP7 & VP8 & VP9 & VB1 & VB2 & VB3 & VB4 \\
\hline Notice - Consent Forms & $x$ & & & & & & & & & \\
\hline Genetic sample & $x$ & & & & & & & & & \\
\hline Heart Rate Variability & $x$ & $x$ & $x$ & $x$ & $x$ & $x$ & & & & \\
\hline Blood sample & $x$ & & $x$ & & $x$ & & $x$ & & & \\
\hline Hair sample & $x$ & & & & & & $x$ & & & \\
\hline Questionnaires booklet & $\mathrm{x}$ & $x$ & $\mathrm{x}$ & $\mathrm{x}$ & $\mathrm{x}$ & $\mathrm{x}$ & $x$ & $x$ & $\mathrm{x}$ & $\mathrm{x}$ \\
\hline
\end{tabular}

Figure 1 Synopsis of study design. (Top) Study timeline for each participant and (bottom) details of visit requirements. Each participant will have to attend 10 visits from their first trimester of pregnancy until 12 months postdelivery. VB, postbirth visits 48 hours, 2, 6 and 12 months postdelivery; VI: inclusion visit; VP, pregnancy visits from the 5th month to the 9th month of pregnancy.

Table 1 Summary of psychological and psychopathological questionnaires

\begin{tabular}{|c|c|c|c|c|c|}
\hline & & Pregnancy & & Postbirth & \\
\hline & & Inclusion visit & VP5 to VP9 & VB1 & $\begin{array}{l}\text { VB2, VB3 and } \\
\text { VB4 }\end{array}$ \\
\hline Pathological & Symptom Checklist 90 & $x$ & & & \\
\hline & State-Trait Anxiety Inventory & $x$ & & x (state) & x (state) \\
\hline & Edinburgh Postnatal Depression Scale & $x$ & & $x$ & $x$ \\
\hline & PTSD Checklist for DMS-5 & $x$ & & & $x$ \\
\hline & Profile of Mood Scale & $x$ & $\mathrm{x}$ & & \\
\hline & Questionnaire to detect the risk of PND & $x$ & $x$ & & \\
\hline & Traumatic Event Scale & & & $x$ & \\
\hline & $\begin{array}{l}\text { Peritraumatic Dissociative Experiences } \\
\text { Questionnaire }\end{array}$ & & & $\mathrm{x}$ & \\
\hline & Traumatic Delivery Impact & & & $x$ & \\
\hline & Maternal Self-Esteem & & & & $x$ \\
\hline Personality & Temperament and Character Inventory & $x$ & & & \\
\hline Positive psychology & Freiburg Mindfulness Inventory & $x$ & & & \\
\hline & $\begin{array}{l}\text { Warwick Edinburgh Mental Well-Being } \\
\text { Scale }\end{array}$ & $x$ & & & \\
\hline & Prenatal Antenatal Inventory & $x$ & $x$ & & \\
\hline & $\begin{array}{l}\text { Multidimensional Scale of Perceived } \\
\text { Social Support }\end{array}$ & $\mathrm{x}$ & $\mathrm{x}$ & $x$ & $x$ \\
\hline & Labour Agentry Scale & & & $x$ & \\
\hline VAS & Professional stress & $x$ & $\mathrm{x}$ & & \\
\hline & Personal stress & $x$ & $x$ & & \\
\hline & Sleep quality & $x$ & $\mathrm{x}$ & & \\
\hline & Delivery Anticipatory Stress & $x$ & $x$ & & \\
\hline & Medical staff support during delivery & & & $x$ & \\
\hline
\end{tabular}

Three psychological aspects are studied: personality, psychopathology and positive psychology. VAS aim to determine stress level.

DSM, Diagnostic and Statistical Manual of Mental Disorders; PND, postnatal depression; VAS, visual analogue scale, VP 
Table 2 Summary of gene polymorphism analysis

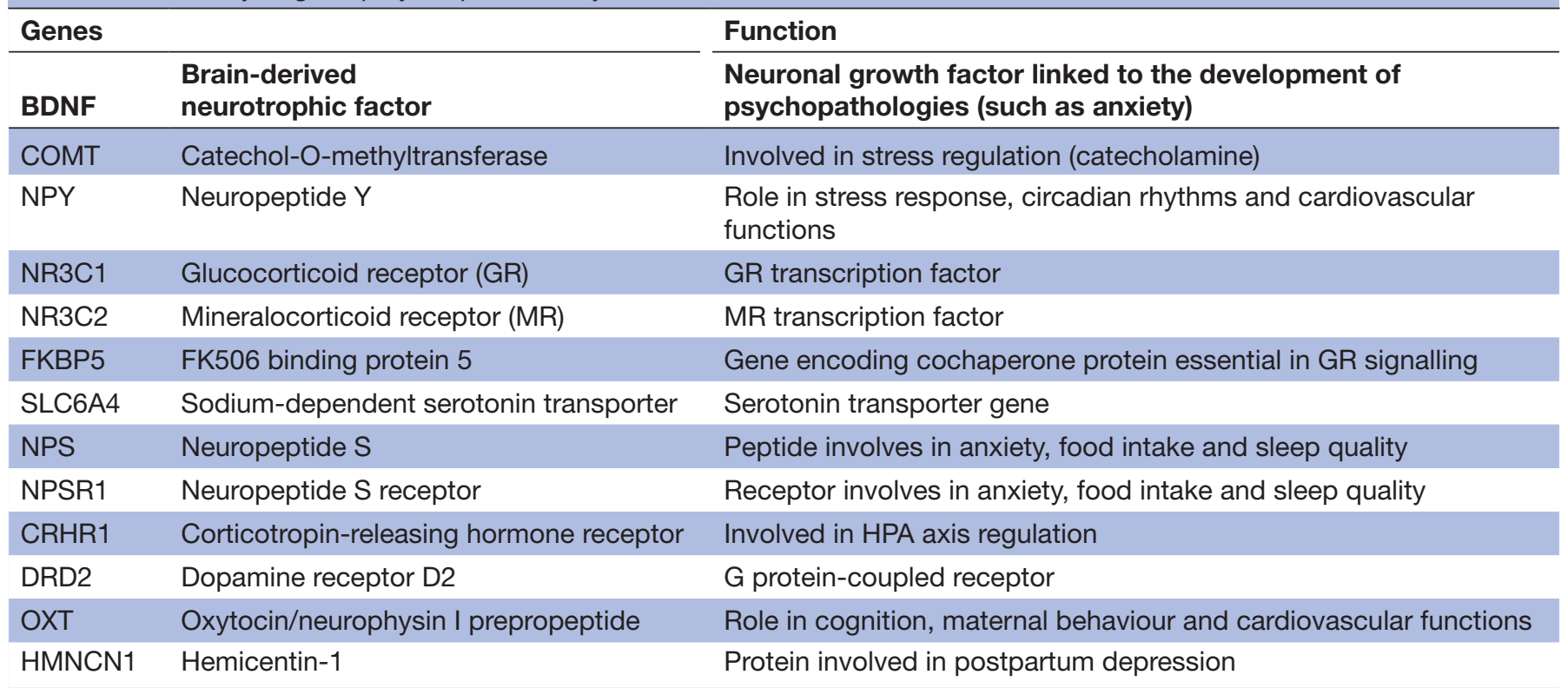

The chosen single nucleotid polymorphism (SNP) aim to characterise vulnerability and severity of PND as well as stress sensitivity.

HPA, hypothalamo-pituitary-adrenal axis; PND, postnatal depression.

The secondary outcomes will contribute to the understanding of the stress mechanisms involved throughout the pregnancy:

i. Biological stress: GABA, thiobarbituric acid reactive substances (byproducts of lipid peroxydation), inflammatory cytokines, cortisol and the endobiogenic index.

ii. Physiological stress: measurement of HRV to determine the activation of the autonomous nervous system.

iii. Psychological stress: perceived stress, social support and life quality.

The exploratory objectives will include the study of genetic polymorphisms linked to stress vulnerability (see table 2) and central functioning (BDNF and eight iso-prostaglandine F2a).

\section{Material}

Heart rate variability

HRV will be measured in order to assess the reactivity of the autonomic nervous system. HRV will be recorded on a monthly basis using the CODESNA Physioner system. Heart rate, HRV components (high frequency (HF); low frequency (LF) and LF/HF ratio) will be examined as described by the Task Force. ${ }^{39}$ The measurement of HRV will be conducted in sitting and resting participant.

\section{Questionnaires}

The psychological assessment will encompass pathological, personality and positive psychology aspects (for details, see table 1). Stress levels will be assessed using visual analogue scales. All the selected questionnaires are self-reporting questionnaires that have been validated in French and have demonstrated good psychometric properties. Ideally, all of these assessments should be performed before any potentially stressful exam.

\section{Blood, hair and genetic samples}

All sampling will be conducted according to the standard operating procedures described by the sponsor and in accordance with local procedures. Hair, blood and genetic samples will be conditioned locally and stored at room temperature or at $-20^{\circ} \mathrm{C}$. All biological materials will then be transferred for analysis to the laboratories (Biolabs or IRBA) by a professional delivery company. Blood samples will be used for the analysis of all the biological parameters, except cortisol, which will be assessed using hair.

\section{Statistics}

Number of participants needed

Several lines of evidence have been used in order to determine the number of participants required.

1. Maternity activities: over 2500 births/year in the CHU Pellegrin and CHR Mercy, 1000 births/year in GH Diaconesses Croix St Simon and up to 200 medically assisted procreations in the CHU Estaing.

2. In the general population, prevalence of PND is $20 \%$; therefore, if we include 260 subjects, 52 should be diagnosed with PND. Among these, $5 \%$ of the mothers-to-be might discontinue the Stress protection for postnatal dEpREssioN prevention (SERENE) project before completion, so 42 participants with PND should remain.

3. The psychometric tools used in the general population show that it is possible to distinguish between mindful 
and less mindful subjects within a sample of 20 subjects (Friburg Mindful Inventory cut-off score above 37). However, since mindfulness has never been assessed in pregnant women, no threshold between high and low mindfulness has been determined in such a population.

4. Given the prevalence of stress in the general population $(30 \%)$, we expect to get 78 stressed subjects among the 260 participants included.

Therefore, we aim to recruit 260 participants, which represents less than $5 \%$ of the total pregnancies followed in the five centres in 1 year.

\section{General design}

Central tendency and the variability of the continuous variables will be described depending on whether or not they follow a normal distribution (using the Shapiro-Wilk test). If there is normal distribution, data will be summarised using means and SD, if not medians and ranges will be used. VAS variables will be analysed like ordinal data and described as above.

If required, class gathering into categorical variables will be performed. These variables will be described as absolute values and percentages.

Any variable with more than $10 \%$ missing data will not be analysed.

\section{Analyses for the primary outcome}

A cut-off score of 11 in the EPDS has been shown to be a sensitive and specific threshold for PND emergence. We will use multivariate logistic regression in order to detect predictive factors of PND emergence. Threshold sensitivity in terms of sensitivity, specificity and predictive value will be performed using receiver operating characteristic analysis.

A two-step approach will be conducted. First, a theme reasoned mixed approach will be undertaken to evaluate the weight of each biomarker for PND risk. Four themes have been identified: (1) biography (full-term delivery and life events), (2) psychological resources, (3) biophysiological profiles and (4) genetics. A final model will then be determined using mixed logistic regression from the variables selected from the previous step.

\section{Analyses for the secondary and exploratory outcomes}

The role of each biomarker and psychological factor in PND will be compared between the two groups (PND and non-PND) using an analysis of variance factorial analysis. Life events during the pregnancy and the subject's status (civilian, military or spouse of force personnel) will be considered. PND risk factors linked to difficult labour/delivery will be controlled.

Finally, a composite index taking all the relevant biomarkers together will be computed in order to have a single predictive variable for the risk of developing PND.

\section{Data collection and management}

In order to ensure the quality of the data collected and to promote participant retention, an assistant will be in charge of conducting the project according to the protocol in each centre involved in the SERENE project. The study will be implemented and conducted by the clinical research assistant (CRA) coordinator of the Direction Centrale du Service de Santé des Armées (DCSSA) in accordance with to the monitoring plan. As much as possible, the CRA will ensure the quality of the collected data in each centre in order to minimise missing data.

Personal information, data entry, coding, security and storage are processed in compliance with the Data Processing, Data Files and Individual Liberties Act of 6 January 1978.

\section{Ethics and dissemination}

The study poses little to no risk to the participants or their infants and does not interfere with the typical care received during pregnancy. This protocol will certainly detect some episodes of depression; such an event will be communicated to the caregivers or the participant herself in order to set up an adequate alternative care. Study results will be disseminated at national and international conferences and in peer-reviewed journals. The trial findings could also be made available to participants collectively. Any modifications of the protocol will be submitted to the competent authorities (if required) and communicated to every relevant party as specified by the French public health code.

All the data will remain the property of the sponsor. The final data report will be written by all the associated scientific investigators (as defined in the protocol).

\section{DISCUSSION}

\section{Potential limitations}

We have identified two potential limitations:

i. Given the way none pathological pregnancies are medically monitored in France (mostly outside of hospitals until the last 2 months of pregnancy), it may be difficult to include 260 participants in their first trimester. For this reason, a 'recruiting network' was initiated in each maternity (ie, involvement of doctors doing first trimester ultrasounds and network of independent midwives), which should help to reach this goal.

ii. For the study, participants need to attend 10 visits, which might seem demanding. Furthermore, the last three visits are important since the diagnosis of PND may be made at this time. So, to make sure that participants will complete the protocol, one person (other than the investigators) is in charge of the participants' follow-up in each maternity. 


\section{Trial status}

The study is currently recruiting participants.

\section{Author affiliations}

${ }^{1}$ Maternité GH Diaconesses Croix St Simon, Paris, France

${ }^{2} U n i t e ́$ de Neurophysiologie du Stress, Département Neurosciences et Contraintes Opérationnelles, Institute de Recherche Biomédicale des Armées, Brétigny Sur Orge, France

${ }^{3}$ INSERM 1219, EPS Charles Perrens et Centre de RecherchE, Bordeaux, France

${ }^{4}$ Apemac, University of Lorraine, Vandoeuvre-lès-Nancy, France

${ }^{5}$ Centre Aliénor d'Aquitaine, CHU Pellegrin, University Hospital of Bordeaux,

Bordeaux, France

${ }^{6} \mathrm{CHR}$ Mercy, Regional Hospital of Metz, Metz, France

${ }^{7}$ Department of In-Vitro Fecondation, Gynecologia, CHU Clermont-Ferrand, University Hospital of Clermont-Ferrand, Clermont-Ferrand, France

${ }^{8}$ Université Clermont Auvergne, UMR CNRS 6024, LaPSC0, Physiological and Psychosocial Stress, CHU Clermont-Ferrand, University Hospital of ClermontFerrand, Occupational and Preventive Medicine, Clermont-Ferrand, France

${ }^{9}$ Faculty of Health, Australian Catholic University, Melbourne, Victoria, Australia

Correction notice This article has been corrected since it was first published. Figure 1 has been replaced with a new version.

Contributors MT, DT and AMD were involved in the conception and design of the trial. They also wrote the draft of the article. A-LS-D, DD, ES, TH, EW, J-LP and FD contributed to the refinement of the study protocol and provided expert insight. MT, DF and AMD were responsible for the ethics committee. FC, MB, LC and FT carried out the study in the different maternities. All the authors were involved in final approval of the manuscript.

Funding The SERENE project is funded by the French Defence Procurement Agency (DGA).

Competing interests None declared.

Patient consent Obtained.

Ethics approval Ethical approval has been obtained from the lle de France III Ethics Committee (Hopital Tarnier-Cochin, Paris), France (2016-A00887-44).

Provenance and peer review Not commissioned; externally peer reviewed.

Data sharing statement Since the manuscript is a protocol paper, there is no unpublished data. The original protocol (version $2-25$ July 2016) for this study is available by contacting the corresponding author via email.

Author note The study sponsor is the Direction centrale du service de santé des armées (DCSSA - Fort neuf de Vincennes, Cours des maréchaux, 75614 Paris Cedex 12). The coordinator, clinical research associate coordinator and the 'Bureau de gestion de la recherche clinique' are in charge of the study logistics: quality of the data collected in the CRF, creation of input masks, computerisation and quality control of the data. Since the study does not involve the use of product or medication, and only represents minor risks and constraints, no data monitoring committee has been formed.

Open Access This is an Open Access article distributed in accordance with the Creative Commons Attribution Non Commercial (CC BY-NC 4.0) license, which permits others to distribute, remix, adapt, build upon this work non-commercially, and license their derivative works on different terms, provided the original work is properly cited and the use is non-commercial. See: http://creativecommons.org/ licenses/by-nc/4.0/

(C) Article author(s) (or their employer(s) unless otherwise stated in the text of the article) 2018. All rights reserved. No commercial use is permitted unless otherwise expressly granted.

\section{REFERENCES}

1. Sutter-dallay AL GNE. Concept de psychiatrie périnatale, histoire, applications, limites. Psychiatrie/Pédopsychiatrie. Vol. B-20. Paris: Elsevier Masson SAS, 2010.

2. Heim A HN, Philippe HJ, Nuss P, et al. Troubles psychiatriques du post-partum. EMC Obstétrique. Paris: Elsevier Masson SAS, 2000.

3. https://cmha.ca/fr/documents/la-depression-postnatale

4. Gibson J, McKenzie-McHarg K, Shakespeare J, et al. A systematic review of studies validating the Edinburgh Postnatal Depression
Scale in antepartum and postpartum women. Acta Psychiatr Scand 2009;119:350-64.

5. Cox JL, Murray D, Chapman G. A controlled study of the onset, duration and prevalence of postnatal depression. Br J Psychiatry 1993;163:27-31.

6. O'Hara MW, Schlechte JA, Lewis DA, et al. Controlled prospective study of postpartum mood disorders: psychological, environmental, and hormonal variables. J Abnorm Psychol 1991;100:63-73.

7. Cooper PJ, Murray L. Postnatal depression. BMJ 1998;316:1884-6.

8. Sharma V, Mazmanian D. The DSM-5 peripartum specifier: prospects and pitfalls. Arch Womens Ment Health 2014;17:171-3.

9. Association, A.P. Diagnostic and statistical manual of mental disorders. Paris, Masson: Texte révisé, 2003.

10. OMS. CIM10 classification statistique internationale des maladies et des problèmes de Santé connexes, 1993.

11. Moraes GP, Lorenzo L, Pontes GA, et al. Screening and diagnosing postpartum depression: when and how? Trends Psychiatry Psychother 2017;39:54-61.

12. CES. The hormone withdrawal hypothesis of postpartum depression: a translational approach. USA: University of lowa, 2011.

13. Galea LA, Wide JK, Barr AM. Estradiol alleviates depressive-like symptoms in a novel animal model of post-partum depression. Behav Brain Res 2001;122:1-9.

14. Jolley SN, Elmore S, Barnard KE, et al. Dysregulation of the hypothalamic-pituitary-adrenal axis in postpartum depression. Biol Res Nurs 2007;8:210-22.

15. Meinlschmidt G, Martin C, Neumann ID, et al. Maternal cortisol in late pregnancy and hypothalamic-pituitary-adrenal reactivity to psychosocial stress postpartum in women. Stress 2010;13:163-71.

16. Rosa CE, Soares JC, Figueiredo FP, et al. Glutamatergic and neural dysfunction in postpartum depression using magnetic resonance spectroscopy. Psychiatry Res 2017;265:18-25.

17. Silverman ME, Loudon $\mathrm{H}$, Safier M, et al. Neural dysfunction in postpartum depression: an fMRI pilot study. CNS Spectr 2007;12:853-62.

18. Maguire J, Mody I. GABA(A)R plasticity during pregnancy: relevance to postpartum depression. Neuron 2008;59:207-13.

19. Grases G, Colom MA, Fernandez RA, et al. Evidence of higher oxidative status in depression and anxiety. Oxid Med Cell Longev 2014;2014:1-5

20. Maes M, Ombelet W, Verkerk R, et al. Effects of pregnancy and delivery on the availability of plasma tryptophan to the brain: relationships to delivery-induced immune activation and early post-partum anxiety and depression. Psychol Med 2001;31:847-58.

21. Zonana J, Gorman JM. The neurobiology of postpartum depression. CNS Spectr 2005;10:792-805.

22. Zelkowitz P, Gold I, Feeley N, et al. Psychosocial stress moderates the relationships between oxytocin, perinatal depression, and maternal behavior. Horm Behav 2014;66:351-60.

23. Christian LM, Mitchell AM, Gillespie SL, et al. Serum brain-derived neurotrophic factor (BDNF) across pregnancy and postpartum: Associations with race, depressive symptoms, and low birth weight. Psychoneuroendocrinology 2016;74:69-76.

24. Corwin EJ, Brownstead J, Barton N, et al. The impact of fatigue on the development of postpartum depression. J Obstet Gynecol Neonatal Nurs 2005;34:577-86.

25. Raison CL, Capuron L, Miller AH. Cytokines sing the blues: inflammation and the pathogenesis of depression. Trends Immunol 2006;27:24-31.

26. Treloar SA, Martin NG, Bucholz KK, et al. Genetic influences on postnatal depressive symptoms: findings from an Australian twin sample. Psychol Med 1999;29:645-54.

27. Mitchell C, Notterman D, Brooks-Gunn J, et al. Role of mother's genes and environment in postpartum depression. Proc Natl Acad Sci U S A 2011;108:8189-93.

28. Gress-Smith JL, Luecken LJ, Lemery-Chalfant K, et al. Postpartum depression prevalence and impact on infant health, weight, and sleep in low-income and ethnic minority women and infants. Matern Child Health J 2012;16:887-93.

29. O'hara MW, Swain AM. Rates and risk of postpartum depressiona meta-analysis. International Review of Psychiatry 1996;8:37-54.

30. Halbreich $U$, Karkun S. Cross-cultural and social diversity of prevalence of postpartum depression and depressive symptoms. $J$ Affect Disord 2006;91:97-111.

31. Brown KW, Ryan RM. The benefits of being present: mindfulness and its role in psychological well-being. J Pers Soc Psychol 2003;84:822-48. 
32. Faisal-Cury A, Tedesco JJ, Kahhale S, et al. Postpartum depression: in relation to life events and patterns of coping. Arch Womens Ment Health 2004;7:123-31.

33. Kabat-Zinn J. Full catastrophe living: using the wisdom of your body mind to face stress, pain, illness. New York: UD connection, 1990.

34. Walach $\mathrm{H}$, Buttenmüller $\mathrm{V}$, Buttenmüller $\mathrm{V}$, et al. Measuring mindfulness - the Freiburg Mindfulness Inventory (FMI). Personality and Individual Differences 2006;40:1543-55.

35. Trousselard M, Cian C BP. Escape from a diving submarine: impacts of mindfulness differences on physio-biological responses, and cognitive performances. Human performance enhancement for nato military operations (sciences, technology, and ethics), in Human factors \& medicine panel symposium (HFM-181). Sofia, 2009.
36. Kabat-Zinn J. Mindfulness-based interventions in context: past, present, and future. Science and Practice 2003;10:144-56.

37. Grossman P, Niemann L, Schmidt S, et al. Mindfulness-based stress reduction and health benefits. A meta-analysis. J Psychosom Res 2004;57:35-43.

38. Cox JL, Holden JM, Sagovsky R. Detection of postnatal depression. Development of the 10-item Edinburgh Postnatal Depression Scale. Br J Psychiatry 1987;150:782-6.

39. Malik M. Heart rate variability: standards of measurement, physiological interpretation and clinical use. Task Force of the European Society of Cardiology and the North American Society of Pacing and Electrophysiology. Circulation 1996;93:1043-65. 